Chinese Journal of Organic Chemistry

\title{
铜催化一锅法合成吡咯[1,2-a]喹喔啉衍生物
}

蒋增强张杰童耀史雪松苗大状韩世清*

(南京工业大学生物与制药工程学院 南京 211816)

\begin{abstract}
摘要 建立了一种简单实用、经济高效的以邻碘苯胺、吡咯甲醛为起始原料, 廉价的 $\mathrm{CuI}$ 为催化剂, 价廉、易获得的 2,2-联吡啶为配体, $110{ }^{\circ} \mathrm{C}$ 条件下一锅法合成吡咯[1,2- $a$ ]喹喔啉类衍生物的催化体系，得到中等到优良产率. 本方法具 有催化剂廉价易得、操作简便、合成效率高的优点.
\end{abstract}

关键词 一锅法合成; 吡咯 $[1,2-a]$ 喹喔啉; 铜催化

\section{One-Pot Synthesis of Pyrrolo[1,2-a]quinoxaline Derivatives through Copper-Catalyzed Cascade Reactions}

\author{
Jiang, Zengqiang Zhang, Jie Tong, Yao Shi, Xuesong \\ Miao, Dazhuang Han, Shiqing* \\ (College of Biotechnology and Pharmaceutical Engineering, Nanjing University of Technology, Nanjing 211816)
}

\begin{abstract}
A simple, efficient, inexpensive one-pot method for the pyrrolo[1,2- $a$ ]quinoxaline derivatives synthesized from 2-formylazoles and $o$-aminoiodoarenes was developed by using cheap $\mathrm{CuI}$ as catalyst, inexpensive and readily available 2,2'-bipyridine as ligand at $110{ }^{\circ} \mathrm{C}$. The reaction provides an efficient protocol to a series of pyrrolo[1,2-a]quinoxaline derivatives in good to high yields, and benefits of readily available catalyst and synthesis of high efficiency.
\end{abstract}

Keywords one-pot synthesis; pyrrolo[1,2- $a$ ]quinoxalines; copper-catalyst

吡咯 $[1,2-a]$ 喹喔啉是很多天然产物分子及生物活性 分子的骨架结构, 广泛存在于生物化学、药物化学以及 材料科学等领域 ${ }^{[1 ~ 3]}$. 因此对吡咯 $[1,2-a]$ 喹喔啉这一骨 架结构的研究, 引起了科学家们的高度关注. Cheese$\operatorname{man}$ 小组 ${ }^{[4]}$ 首次报道了合成吡咯 $[1,2-a]$ 喹呿啉的新方法, 该反应体系需经两步合成吡咯 $[1,2-a]$ 喹喔啉, 反应时间 长, 反应过程繁琐; 随着利用金属催化剂来构建 $\mathrm{C}-\mathrm{N}$ 键的 Ullmann 反应研究的逐渐深入、其中铜催化剂 ${ }^{[5]}$ 以 其廉价易得、低毒及催化活性高的优点最受关注. 2008 年, 马大为小组 ${ }^{[6]}$ 利用 $\mathrm{CuI}$ 催化偶联反应合成吡咯 $[1,2-a]$ 喹喔啉衍生物, 该反应过程需要三氟乙酰基的水 解和分子间氨基化合物的形成; 2010 年, Reeves 小组 ${ }^{[7]}$ 报道了利用铜催化合成吡咯 [1,2- $a$ ]喹喔啉的反应, 该体 系避免了复杂底物的使用, 但反应需要较高的温度 $(130$ ${ }^{\circ} \mathrm{C}$ ), 同时反应中使用的配体结构复杂, 价格昂贵, 不易 获得; Valerie 小组 ${ }^{[8]}$ 利用铁催化芳硝基的还原和乙醇的
需氧氧化来构建吡咯 $[1,2-a]$ 喹喔啉衍生物, 该体系需要 邻位带有吡咯取代基的硝基苯这一复杂底物的参与，底 物适用范围不够广泛; 2013 年, 马晨小组 ${ }^{[9]}$ 报道了在空 气中利用醋酸铜催化 $N$-磺酰基-2-卤苯胺类化合物与 2-(氯甲基)- $1 H$-苯并 $[d]$ 咪唑类化合物合成吡咯 $[1,2-a]$ 喹 喔啉类衍生物，该体系只适用于带有磺酰基的卤苯胺类 化合物, 限制了底物的适用范围, 同时反应需要强碱 $\left(\mathrm{Cs}_{2} \mathrm{CO}_{3}\right)$ 的参与.

我们课题组 ${ }^{[10]}$ 在前期工作的研究基础上, 发现利 用邻碘苯胺与吡咯甲醛在铜催化的促进下、在较温和条 件下反应，可以一步合成此类化合物. 本文报道一种反 应条件温和、经济高效的铜催化一锅法合成吡咯 $[1,2-a]$ 喹喔啉类衍生物反应体系: 在 DMF 中使用 $\mathrm{CuI}$ 为催化 剂, 价廉易得的 2,2-联吡啶为配体, $\mathrm{K}_{3} \mathrm{PO}_{4}$ 为碱性介质, $110{ }^{\circ} \mathrm{C}$ 条件下催化邻碘苯胺与吡咯甲醛串联反应合成吡 咯[1,2- $a]$ 喹喔啉, 获得了满意的结果.

\footnotetext{
*E-mail: hanshiqing@njut.edu.cn

Received March 10, 2014; revised April 17, 2014; published online May 6, 2014.

Project supported by the National Natural Science Foundation of China (No. 21072095), the Graduate Student Innovation Project in Jiangsu Province (No. CXLX13_433).

国家自然科学基金(No. 21072095)和江苏省研究生创新工程(No. CXLX13_433)资助项目.
} 


\section{1 结果与讨论}

\section{1 最佳反应条件建立}

首先以邻碘苯胺与吡咯甲醛为模型反应底物, 在 $\mathrm{N}_{2}$ 保护条件下考察了各类铜盐催化剂、配体、碱对反应 结果的影响, 结果见表 1. 一价铜盐的催化效果优于二 价铜盐(表 1, Entries 1 5), 其中 $\mathrm{CuI}$ 催化效果最好. 之 后, 我们考察了各种碱对反应的影响, 在被考察的碱中, $\mathrm{K}_{3} \mathrm{PO}_{4}$ 得到了 $55.6 \%$ 的收率，而其它碱虽有一定效果， 但与 $\mathrm{K}_{3} \mathrm{PO}_{4}$ 相比结果都不太理想(表 1, Entries 6 9), 因 此后续反应我们均以 $\mathrm{K}_{3} \mathrm{PO}_{4}$ 作为最有效的碱来参与反 应; 使用其他溶剂如 DMSO、甲苯、乙腈、1,4-二氧六环 都不利反应进行, 原料转化率下降(表 1, Entries 10 13). 从表 1 可以看出, 所选用的六种结构简单、价廉的 配体中(表 1, Entries 13 18)，2,2-联吡啶的催化效果最 好. 最后我们考察了温度和时间对该反应的影响, 尝试 提高温度到 $110{ }^{\circ} \mathrm{C}$, 产率升高到 78.6\%(表 1, Entry 19), 温度升高到 $130{ }^{\circ} \mathrm{C}$, 产率略有下降(表 1, Entry 20). 条件 对照试验表明反应在空气中进行时反应几乎不发生, $\mathrm{N}_{2}$ 保护是必要的(表 1, Entry 22).

\section{2 铜催化不同底物合成吡咯[1,2-a]喹喔啉类衍生物}

得到 $\mathrm{CuI}$ 催化条件下邻碘苯胺与吡咯甲醛反应合成 吡咯[1,2-a]喹喔啉的最佳条件(表 1, Entry 19)后, 我们进
一步考查此催化体系的普适性(表 2). 从表 2 可知: 催化 体系对于各种 2-碘芳胺和 2-吡咯甲醛均具有较好的催 化活性, 对于大部分底物均能以较好收率专一地生成相 应目标产物. 首先我们将各种不同取代基的卤代芳胺与 吡咯甲醛进行反应. 如表 2 所示，一些杂环卤代芳胺如 2-氨基-3-碘吡啶和 2-吡咯甲醛能得到较好产率(表 2, Entry 3). 此外含有吸电子基团和供电子基团的 2-碘苯 胺与吡咯甲醛反应无显著取代基效应，都能得到相应的 吡咯 $[1,2-a]$ 喹喔啉衍生物，产率从 59.8\%至 78.6\%(表 2, Entries 1 5); 其次为了扩大这种方法的底物适用范围, 我们考察了一系列的取代 2-吡咯甲醛与邻碘苯胺反应. 实验表明，含不同取代基的 2-吡咯甲醛仍旧表现出良好 的活性，该反应条件不仅适用于富电子的吡咯甲醛(表 2, Entries 6 9), 还适用于一些缺电子的吡咯甲醛(表 2, Entries 10,11). 我们还发现该催化体系对于咪唑甲醛和 吡唑甲醛同样适用(表 2, Entries 12１6), 其中咪唑环对 位连有供电子基团 $\left(\mathrm{CH}_{3}\right.$ 和苯环 $)$ 取代的醛 $(\mathbf{2} \mathbf{i}, 2 \mathbf{j})$ 反应效 果优于连有吸电子基团 $(\mathrm{Cl})$ 的醛(2k).

\section{2 结论}

研究了在 DMF 溶剂中, 使用廉价的 CuI, 结构简单 易得的 2,2-联吡啶配体，利用邻卤芳胺与吡咯甲醛一锅 法合成吡咯 $[1,2-a]$ 喹喔啉衍生物的体系. 该催化体系操

表 1 反应条件篮选和优化 ${ }^{a}$

Table 1 Screening and optimization of the reaction conditions

\begin{tabular}{|c|c|c|c|c|c|c|c|}
\hline Entry & Catalyst (equiv.) & Base (equiv.) & Solvent (mL) & Ligand (equiv.) & $t /{ }^{\circ} \mathrm{C}$ & Time/h & Yield $^{b} / \%$ \\
\hline 1 & $\mathrm{CuI}(0.1)$ & $\mathrm{K}_{3} \mathrm{PO}_{4}(3.0)$ & DMF (2.0) & $L$-Pro $(0.2)$ & 90 & 24 & 55.6 \\
\hline 2 & $\mathrm{CuBr}(0.1)$ & $\mathrm{K}_{3} \mathrm{PO}_{4}(3.0)$ & DMF (2.0) & $L$-Pro $(0.2)$ & 90 & 24 & 38.3 \\
\hline 3 & $\mathrm{CuCl}(0.1)$ & $\mathrm{K}_{3} \mathrm{PO}_{4}(3.0)$ & DMF (2.0) & $L$-Pro $(0.2)$ & 90 & 24 & 32.5 \\
\hline 4 & $\mathrm{Cu}_{2} \mathrm{O}(0.1)$ & $\mathrm{K}_{3} \mathrm{PO}_{4}(3.0)$ & DMF (2.0) & $L$-Pro $(0.2)$ & 90 & 24 & 38.9 \\
\hline 5 & $\mathrm{Cu}_{2} \mathrm{Cl}(0.1)$ & $\mathrm{K}_{3} \mathrm{PO}_{4}(3.0)$ & DMF (2.0) & $L$-Pro $(0.2)$ & 90 & 24 & 14.1 \\
\hline 6 & $\mathrm{CuI}(0.1)$ & $\mathrm{K}_{2} \mathrm{CO}_{3}(3.0)$ & DMF (2.0) & $L$-Pro $(0.2)$ & 90 & 24 & 16.0 \\
\hline 7 & $\mathrm{CuI}(0.1)$ & $\mathrm{KOH}(3.0)$ & DMF (2.0) & $L$-Pro $(0.2)$ & 90 & 24 & 11.4 \\
\hline 8 & $\mathrm{CuI}(0.1)$ & $\mathrm{CS}_{2} \mathrm{CO}_{3}(3.0)$ & DMF (2.0) & $L$-Pro $(0.2)$ & 90 & 24 & 32.5 \\
\hline 9 & $\mathrm{CuI}(0.1)$ & $\mathrm{Ag}_{2} \mathrm{CO}_{3}(3.0)$ & DMF (2.0) & $L$-Pro $(0.2)$ & 90 & 24 & 43.9 \\
\hline 10 & $\mathrm{CuI}(0.1)$ & $\mathrm{K}_{3} \mathrm{PO}_{4}(3.0)$ & DMSO (2.0) & $L$-Pro $(0.2)$ & 90 & 24 & 49.4 \\
\hline 11 & $\mathrm{CuI}(0.1)$ & $\mathrm{K}_{3} \mathrm{PO}_{4}(3.0)$ & Toluene (2.0) & $L$-Pro $(0.2)$ & 90 & 24 & 50.5 \\
\hline 12 & $\mathrm{CuI}(0.1)$ & $\mathrm{K}_{3} \mathrm{PO}_{4}(3.0)$ & $\mathrm{CH}_{3} \mathrm{CN}(2.0)$ & $L$-Pro $(0.2)$ & 90 & 24 & 26.7 \\
\hline 13 & $\mathrm{CuI}(0.1)$ & $\mathrm{K}_{3} \mathrm{PO}_{4}(3.0)$ & 1,4-Dioxane (2.0) & $L$-Pro $(0.2)$ & 90 & 24 & 26.9 \\
\hline 14 & $\mathrm{CuI}(0.1)$ & $\mathrm{K}_{3} \mathrm{PO}_{4}(3.0)$ & $\operatorname{DMF}(2.0)$ & 8-Hydroxyquinoline $(0.2)$ & 90 & 24 & 45.8 \\
\hline 15 & $\mathrm{CuI}(0.1)$ & $\mathrm{K}_{3} \mathrm{PO}_{4}(3.0)$ & DMF (2.0) & 1,10-Phenanthroline $(0.2)$ & 90 & 24 & 57.8 \\
\hline 16 & $\mathrm{CuI}(0.1)$ & $\mathrm{K}_{3} \mathrm{PO}_{4}(3.0)$ & $\operatorname{DMF}(2.0)$ & 1,2-Ethanediamine $(0.2)$ & 90 & 24 & Trace \\
\hline 17 & $\mathrm{CuI}(0.1)$ & $\mathrm{K}_{3} \mathrm{PO}_{4}(3.0)$ & DMF (2.0) & 1,2-Ethanediol $(0.2)$ & 90 & 24 & Trace \\
\hline 18 & $\mathrm{CuI}(0.1)$ & $\mathrm{K}_{3} \mathrm{PO}_{4}(3.0)$ & $\operatorname{DMF}(2.0)$ & 2,2-Dipyridyl (0.2) & 90 & 24 & 61.7 \\
\hline 19 & $\mathrm{CuI}(\mathbf{0 . 1})$ & $\mathrm{K}_{3} \mathrm{PO}_{4}(3.0)$ & DMF (2.0) & 2,2-Dipyridyl (0.2) & 110 & 24 & 78.6 \\
\hline 20 & $\mathrm{CuI}(0.1)$ & $\mathrm{K}_{3} \mathrm{PO}_{4}(3.0)$ & DMF (2.0) & 2,2-Dipyridyl (0.2) & 130 & 24 & 64.2 \\
\hline 21 & $\mathrm{CuI}(0.1)$ & $\mathrm{K}_{3} \mathrm{PO}_{4}(3.0)$ & DMF (2.0) & 2,2-Dipyridyl (0.2) & 110 & 12 & 36.4 \\
\hline 22 & $\mathrm{CuI}(0.1)$ & $\mathrm{K}_{3} \mathrm{PO}_{4}(3.0)$ & $\operatorname{DMF}(2.0)$ & 2,2-Dipyridyl (0.2) & 110 & 24 & Trace $^{c}$ \\
\hline
\end{tabular}

反应条件: 2-碘苯胺 $(0.215 \mathrm{mmol})$, 吡咯甲醛 $(0.215 \mathrm{mmol})$, 铜盐 $(0.0215 \mathrm{mmol})$, 配体 $(0.043 \mathrm{mmol})$, 碱 $(0.645 \mathrm{mmol})$, 溶剂 $(2.0 \mathrm{~mL}) ;{ }^{b}$ 分离产率; ${ }^{c}$ 空气中. 
表 2 吡咯 $[1,2-a]$ 喹喔啉类衍生物的合成 ${ }^{a}$

Table 2 The synthesis of pyrrolo[1,2- $a$ ]quinoxaline derivatives

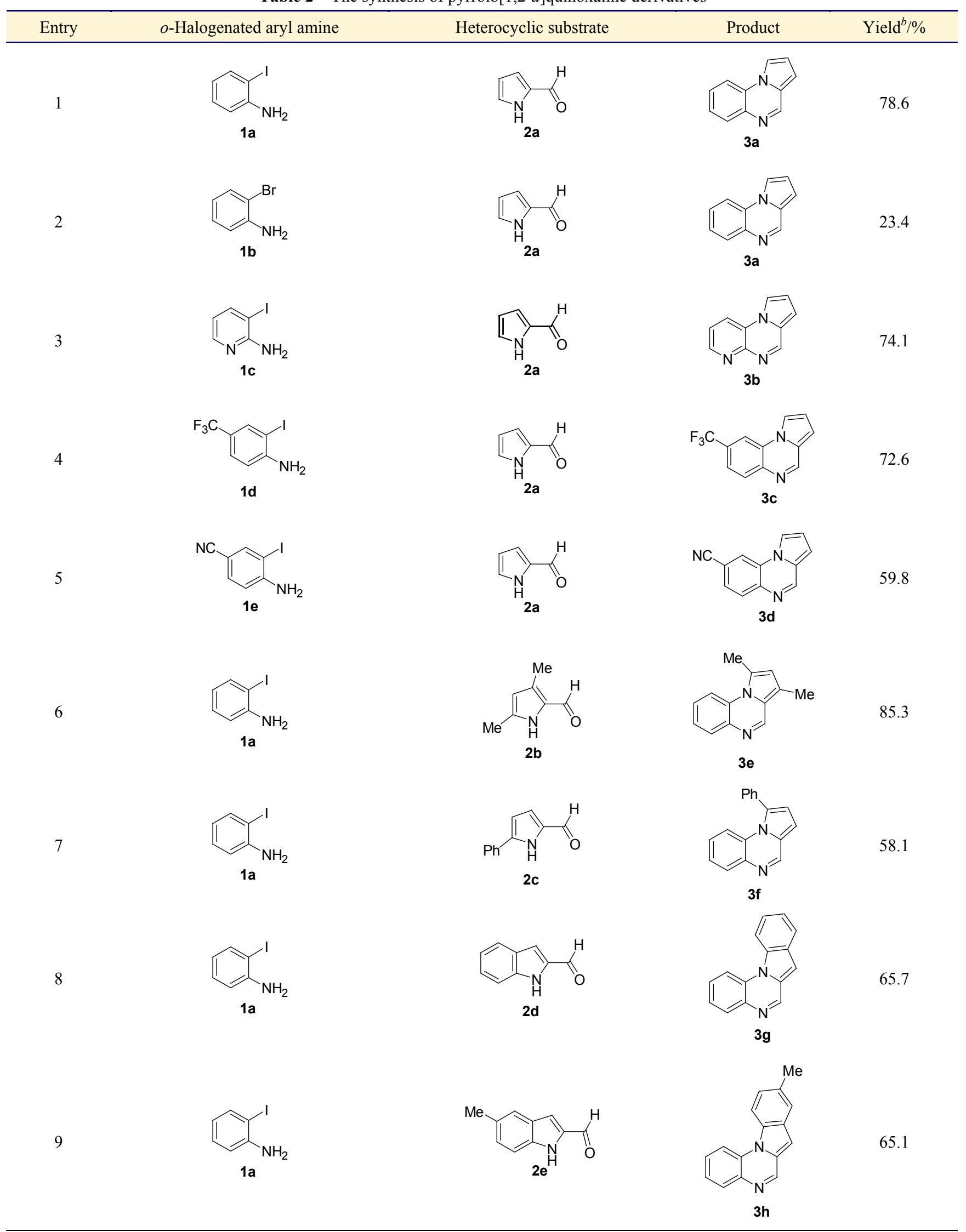




\begin{tabular}{cc}
\hline Entry & $o$-Halogenated aryl \\
\hline 10 &
\end{tabular}

1a

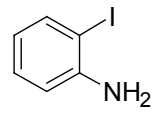

$1 \mathrm{a}$

12

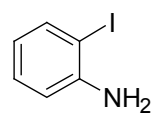

1a

13

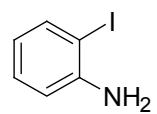

1a

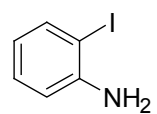

1a

15

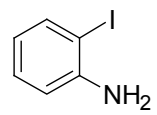

$1 a$

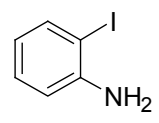

$1 a$
Heterocyclic substrate

$\mathrm{O}_{2} \mathrm{~N}$<smiles>Cc1ccc2[nH]c(C=O)cc2c1</smiles>

$2 f$<smiles>O=Cc1cc2cc(Cl)ccc2[nH]1</smiles>

2g<smiles>O=Cc1ncc[nH]1</smiles>

$2 \mathrm{~h}$<smiles>O=Cc1nc2ccccc2[nH]1</smiles>

2i<smiles>Cc1ccc2[nH]c(C=O)nc2c1</smiles>

2j<smiles>O=Cc1nc2cc(Cl)ccc2[nH]1</smiles>

2k<smiles>Cc1cc(C=O)[nH]n1</smiles>

Product

Yield $^{b} / \%$<smiles>O=[N+]([O-])c1ccc2c(c1)cc1cnc3ccccc3n12</smiles>

$3 \mathbf{i}$<smiles>Clc1ccc2c(c1)cc1cnc3ccccc3n12</smiles>

$3 \mathbf{j}$<smiles>c1ccc2c(c1)ncc1nccn12</smiles>

90.8

80.6

质应条件: 邻碘苯胺 $(0.215 \mathrm{mmol})$, 吡咯甲醛 $(0.215 \mathrm{mmol})$, 磷酸钾 $(0.63 \mathrm{mmol})$, 碘化亚铜 $(0.0215 \mathrm{mmol}), 2,2$-联吡啶 $(0.043 \mathrm{mmol}), \mathrm{DMF}(2.0 \mathrm{~mL}), 110{ }^{\circ} \mathrm{C}, 24$ $\mathrm{h}, \mathrm{N}_{2}$ 保护 $;^{b}$ 分离产率.

作简便，条件温和，副反应少，产物的后处理方便；避

[1,2- $a]$ 喹喔啉衍生物的经济成本.

免了复杂配体的使用和高温反应，从而降低了合成吡咯 


\section{3 实验部分}

\section{1 仪器与试剂}

所用的试剂均为分析纯, 使用前未进行纯化. ${ }^{1} \mathrm{H}$ NMR 和 ${ }^{13} \mathrm{C}$ NMR 用 Bruker DRX-300 测定, 以 TMS 为 内标, $\mathrm{CDCl}_{3}$ 作溶剂; 高分辨质谱使用安捷伦 6520Q-TOF LC/MS 仪器测定, $\mathrm{CH}_{3} \mathrm{OH}$ 作溶剂; 熔点用 WRS-2A 熔点仪测定. 所用的药品和试剂均为市售的分 析纯或化学纯, 除特别说明外, 未经进一步处理.

\section{2 实验方法}

将胺 $\mathbf{1}(0.215 \mathrm{mmol}) 、$ 醛 $\mathbf{2}(0.215 \mathrm{mmol}) 、 \mathrm{CuI}(0.0215$ $\mathrm{mmol})$ 、磷酸钾 $(0.63 \mathrm{mmol}) 、 2,2$-联吡定 $(0.043 \mathrm{mmol})$ 投 入 $10 \mathrm{~mL}$ 茄形瓶中, $\mathrm{N}_{2}$ 气氛中加入 $\mathrm{DMF}(2.0 \mathrm{~mL})$, 油浴 $110{ }^{\circ} \mathrm{C}$, 搅拌反应 $24 \mathrm{~h}$. 自然降至室温, 加入 $10 \mathrm{~mL}$ $\mathrm{H}_{2} \mathrm{O}$, 用 $10 \mathrm{~mL} \times 3$ 乙酸乙酯萃取, 合并乙酸乙酯相, 用 $5 \mathrm{~mL}$ 饱和食盐水洗涤, 有机层经无水硫酸钠干燥, 浓 缩, 上硅胶柱层析分离, 石油醚/乙酸乙酯为洗脱剂得到 目标产物 3, 计算产率. 目标产物通过 ${ }^{1} \mathrm{H} N \mathrm{NMR},{ }^{13} \mathrm{C}$ NMR 和 HRMS 进行结构表征, 其中 $\mathbf{3 h}, \mathbf{3 i}, \mathbf{3} \mathbf{j}, \mathbf{3 m}, \mathbf{3 n}$ 为 新化合物.

吡咯 $[1,2-a]$ 喹喔啉 $(3 a){ }^{[7]}$ : m.p. $131 \sim 132{ }^{\circ} \mathrm{C}$, 白色固 体. ${ }^{1} \mathrm{H}$ NMR $\left(300 \mathrm{MHz}, \mathrm{CDCl}_{3}\right) \delta: 8.79(\mathrm{~s}, 1 \mathrm{H}), 7.95(\mathrm{~d}$, $J=8.0 \mathrm{~Hz}, 1 \mathrm{H}), 7.83(\mathrm{~s}, 1 \mathrm{H}), 7.81(\mathrm{~d}, J=8.2 \mathrm{~Hz}, 1 \mathrm{H})$, $7.50 \sim 7.47(\mathrm{~m}, 1 \mathrm{H}), 7.43 \sim 7.40(\mathrm{~m}, 1 \mathrm{H}), 6.87 \sim 6.85(\mathrm{~m}$, $2 \mathrm{H}) ;{ }^{13} \mathrm{C}$ NMR (75 MHz, $\left.\mathrm{CDCl}_{3}\right) \delta: 145.9,135.8,130.2$, 128.1, 127.8, 126.5, 125.2, 114.2, 114.0, 113.8, 107.3.

吡啶[2,3-e]并吡咯[1,2-a]吡嗪(3b) ${ }^{[7]}$ : m.p. 169 171 ${ }^{\circ} \mathrm{C}$, 浅黄色固体. ${ }^{1} \mathrm{H}$ NMR $\left(300 \mathrm{MHz}, \mathrm{CDCl}_{3}\right) \delta: 9.00$ (s, $1 \mathrm{H}), 8.77 \sim 8.74(\mathrm{~m}, 1 \mathrm{H}), 8.21 \sim 8.19(\mathrm{~m}, 1 \mathrm{H}), 7.97 \sim 7.96$ (m, 1H), $7.45(\mathrm{~d}, J=8.2 \mathrm{~Hz}, 1 \mathrm{H}), 6.96 \sim 6.91(\mathrm{~m}, 2 \mathrm{H}) ;{ }^{13} \mathrm{C}$ NMR $\left(75 \mathrm{MHz}, \mathrm{CDCl}_{3}\right) \delta: 149.2,147.6,146.9,126.4$, 123.9, 122.4, 122.2, 115.6, 115.2, 108.4.

8-(三氟甲基)吡咯 $[1,2-a]$ 喹喔啉(3c) ${ }^{[7]}$ : m.p. 131 $133{ }^{\circ} \mathrm{C}$, 棕褐色固体. ${ }^{1} \mathrm{H}$ NMR (300 MHz, $\left.\mathrm{CDCl}_{3}\right) \delta: 8.85$ (s, $1 \mathrm{H}), 8.09(\mathrm{~s}, 1 \mathrm{H}), 8.04 \sim 8.02(\mathrm{~m}, 1 \mathrm{H}), 7.97 \sim 7.96(\mathrm{~m}$, 1H), $7.67 \sim 7.65(\mathrm{~m}, 1 \mathrm{H}), 6.97 \sim 6.96(\mathrm{~m}, 1 \mathrm{H}), 6.94 \sim 6.92$ $(\mathrm{m}, 1 \mathrm{H}) ;{ }^{13} \mathrm{C}$ NMR $\left(75 \mathrm{MHz}, \mathrm{CDCl}_{3}\right) \delta: 147.7,137.9$, $130.8,129.5,127.8,126.4,122.4,121.6,115.0,114.8$, 111.5, 108.6.

8-甲腈吡咯 $[1,2-a]$ 喹喔啉 $(3 d)^{[7]}$ : m.p. $231 \sim 234{ }^{\circ} \mathrm{C}$, 棕褐色固体. ${ }^{1} \mathrm{H}$ NMR (300 MHz, DMSO- $\left.d_{6}\right) \delta: 9.15$ (s, $1 \mathrm{H}), 9.01(\mathrm{~d}, J=1.5 \mathrm{~Hz}, 1 \mathrm{H}), 8.75 \sim 8.74(\mathrm{~m}, 1 \mathrm{H}), 8.04(\mathrm{~d}$, $J=8.4 \mathrm{~Hz}, 1 \mathrm{H}), 7.94(\mathrm{~d}, J=8.4,1 \mathrm{H}), 7.33 \sim 7.29(\mathrm{~m}, 1 \mathrm{H})$, $7.13 \sim 7.07(\mathrm{~m}, 1 \mathrm{H}) ;{ }^{13} \mathrm{C}$ NMR (75 MHz, DMSO- $\left.d_{6}\right) \delta$ : $147.6,138.3,129.8,129.5,127.3,125.7,117.0,116.8$,
$116.1,114.5,112.5,108.6$.

1,3-二甲基吡咯 $[1,2-a]$ 喹喔啉 (3e $)^{[7]}$ : m.p. $61 \sim 63{ }^{\circ} \mathrm{C}$, 棕褐色固体. ${ }^{1} \mathrm{H}$ NMR $\left(300 \mathrm{MHz}, \mathrm{CDCl}_{3}\right) \delta: 8.63(\mathrm{~s}, 1 \mathrm{H})$, $8.12(\mathrm{~d}, J=7.4 \mathrm{~Hz}, 1 \mathrm{H}), 7.88(\mathrm{~d}, J=7.0 \mathrm{~Hz}, 1 \mathrm{H}), 7.52 \sim$ $7.36(\mathrm{~m}, 2 \mathrm{H}), 6.39(\mathrm{~s}, 1 \mathrm{H}), 2.86(\mathrm{~s}, 3 \mathrm{H}), 2.41(\mathrm{~s}, 3 \mathrm{H}) ;{ }^{13} \mathrm{C}$ NMR $\left(75 \mathrm{MHz}, \mathrm{CDCl}_{3}\right) \delta: 144.2,137.3,130.4,129.7$, 128.1, 126.2, 124.7, 124.4, 117.3, 116.9, 115.3, 17.6, 10.2. 1-苯基吡咯 $[1,2-a]$ 喹喔啉 $(\mathbf{3 f})^{[7]}$ : 蜡状固体. ${ }^{1} \mathrm{H}$ NMR $\left(300 \mathrm{MHz}, \mathrm{CDCl}_{3}\right) \delta: 8.70(\mathrm{~s}, 1 \mathrm{H}), 7.82(\mathrm{~d}, J=8.0 \mathrm{~Hz}$, $1 \mathrm{H}), 7.40 \sim 7.36(\mathrm{~m}, 5 \mathrm{H}), 7.28(\mathrm{~d}, J=8.5 \mathrm{~Hz}, 1 \mathrm{H}), 7.22(\mathrm{t}$, $J=7.7 \mathrm{~Hz}, 1 \mathrm{H}), 6.99$ (t, $J=8.1 \mathrm{~Hz}, 1 \mathrm{H}), 6.83(\mathrm{~d}, J=4.0$ $\mathrm{Hz}, 1 \mathrm{H}), 6.64(\mathrm{~d}, J=4.0 \mathrm{~Hz}, 1 \mathrm{H}) ;{ }^{13} \mathrm{C}$ NMR $(75 \mathrm{MHz}$, $\left.\mathrm{CDCl}_{3}\right) \delta: 146.1,137.2,134.1,132.4,130.0,129.8,129.1$, $128.9,128.8,128.7,128.6,127.8,126.4,125.0,116.7$, 116.6, 107.6.

吲哚 $[1,2-a]$ 喹喔啉 $(\mathbf{3 g})^{[7]}$ : m.p. $111 \sim 113{ }^{\circ} \mathrm{C}$, 橙色固 体. ${ }^{1} \mathrm{H}$ NMR $\left(300 \mathrm{MHz}, \mathrm{CDCl}_{3}\right) \delta: 8.94(\mathrm{~s}, 1 \mathrm{H}), 8.45 \sim$ $8.42(\mathrm{~m}, 2 \mathrm{H}), 8.00 \sim 7.96(\mathrm{~m}, 2 \mathrm{H}), 7.61(\mathrm{t}, J=7.7 \mathrm{~Hz}, 1 \mathrm{H})$, $7.55(\mathrm{t}, J=7.5 \mathrm{~Hz}, 1 \mathrm{H}), 7.46 \sim 7.41(\mathrm{~m}, 2 \mathrm{H}), 7.14(\mathrm{~s}, 1 \mathrm{H})$; ${ }^{13} \mathrm{C}$ NMR $\left(75 \mathrm{MHz}, \mathrm{CDCl}_{3}\right) \delta: 145.2,137.4,131.3,130.2$, $130.0,129.8,129.2,129.0,126.6,126.1,124.6,124.4$, 125.6, 115.5, 107.3.

9-甲基吲哚 $[1,2-a]$ 喹喔啉(3h): m.p. 126 128 ${ }^{\circ} \mathrm{C}$, 粉红色固体. ${ }^{1} \mathrm{H}$ NMR $\left(300 \mathrm{MHz}, \mathrm{CDCl}_{3}\right) \delta: 8.70(\mathrm{~s}, 1 \mathrm{H})$, $8.18(\mathrm{~d}, J=8.3 \mathrm{~Hz}, 1 \mathrm{H}), 7.93$ (d, $J=8.0 \mathrm{~Hz}, 1 \mathrm{H}), 7.63$ (d, $J=8.6 \mathrm{~Hz}, 1 \mathrm{H}), 7.42(\mathrm{t}, J=7.2 \mathrm{~Hz}, 1 \mathrm{H}), 7.34$ (t, $J=7.8$ $\mathrm{Hz}, 1 \mathrm{H}), 6.96 \sim 6.91(\mathrm{~m}, 1 \mathrm{H}), 6.83 \sim 6.80(\mathrm{~m}, 1 \mathrm{H}), 6.78(\mathrm{~s}$, $1 \mathrm{H}), 2.51(\mathrm{~s}, 3 \mathrm{H}) ;{ }^{13} \mathrm{C} \mathrm{NMR}\left(75 \mathrm{MHz}, \mathrm{CDCl}_{3}\right) \delta: 155.7$, $147.4,135.9,130.8,130.4,130.3,130.2,128.6,128.0$, 123.9, 115.8, 115.5, 114.5, 102.3, 100.2, 29.7; HRMS (EI) calcd for $\mathrm{C}_{16} \mathrm{H}_{12} \mathrm{~N}_{2}(\mathrm{M}+\mathrm{H})^{+}$233.1018, found 233.1023.

9-硝基吲哚 [1,2- $a$ ] 喹喔啉(3i): m.p. $139 \sim 141{ }^{\circ} \mathrm{C}$, 白色固体. ${ }^{1} \mathrm{H}$ NMR $\left(300 \mathrm{MHz}, \mathrm{CDCl}_{3}\right) \delta: 8.89(\mathrm{~s}, 1 \mathrm{H})$, 8.34 (d, $J=8.3 \mathrm{~Hz}, 1 \mathrm{H}), 8.26$ (d, $J=8.3 \mathrm{~Hz}, 1 \mathrm{H}), 7.96$ (d, $J=7.8 \mathrm{~Hz}, 1 \mathrm{H}), 7.58(\mathrm{t}, J=7.8 \mathrm{~Hz}, 1 \mathrm{H}), 7.40$ (t, $J=7.7$ $\mathrm{Hz}, 1 \mathrm{H}), 7.28 \sim 7.27$ (br s, 1H), $7.18 \sim 7.16(\mathrm{~m}, 1 \mathrm{H}), 7.03$ (s, $1 \mathrm{H}) ;{ }^{13} \mathrm{C}$ NMR (75 MHz, $\left.\mathrm{CDCl}_{3}\right) \delta: 146.1,137.2,134.1$, $132.4,129.8,129.6,128.9,128.8,128.7,127.7,126.6$, 124.9, 116.8, 116.6, 107.6; HRMS (EI) calcd for $\mathrm{C}_{15} \mathrm{H}_{9} \mathrm{~N}_{3} \mathrm{O}_{2}(\mathrm{M}+\mathrm{H})^{+}$264.0711, found 264.0714.

9-氯基吲哚 $[1,2-a]$ 喹咥啉 (3j): m.p. $121 \sim 124{ }^{\circ} \mathrm{C}$, 白 色固体. ${ }^{1} \mathrm{H}$ NMR $\left(300 \mathrm{MHz}, \mathrm{CDCl}_{3}\right) \delta: 8.94(\mathrm{~s}, 1 \mathrm{H})$, $8.79 \sim 8.75(\mathrm{~m}, 1 \mathrm{H}), 8.64 \sim 8.63(\mathrm{~m}, 1 \mathrm{H}), 8.00(\mathrm{dd}, J=8.4$, $1.5 \mathrm{~Hz}, 2 \mathrm{H}), 7.92(\mathrm{~d}, J=8.4 \mathrm{~Hz}, 2 \mathrm{H}), 7.09 \sim 7.04(\mathrm{~m}, 1 \mathrm{H})$, $6.96 \sim 6.94(\mathrm{~m}, 1 \mathrm{H}) ;{ }^{13} \mathrm{C} \mathrm{NMR}\left(75 \mathrm{MHz}, \mathrm{CDCl}_{3}\right) \delta: 159.8$, 
$157.4,146.0,135.8,130.4,130.3,130.2,128.6,127.8$, 123.9, 115.4, 114.0, 113.3, 109.2, 104.9; HRMS (EI) calcd for $\mathrm{C}_{15} \mathrm{H}_{9} \mathrm{~N}_{2} \mathrm{Cl}(\mathrm{M}+\mathrm{H})^{+}$253.0543, found 253.0543.

咪唑[1,2- $a$ ]喹喔啉(3k) ${ }^{[11]}$ : m.p. 121 $123{ }^{\circ} \mathrm{C}$, 白色 固体. ${ }^{1} \mathrm{H} \mathrm{NMR}\left(300 \mathrm{MHz}, \mathrm{CDCl}_{3}\right) \delta: 9.13(\mathrm{~s}, 1 \mathrm{H}), 8.15 \sim$ $8.13(\mathrm{~m}, 2 \mathrm{H}), 7.98 \sim 7.93(\mathrm{~m}, 1 \mathrm{H}), 7.83(\mathrm{~s}, 1 \mathrm{H}), 7.70 \sim$ $7.66(\mathrm{~m}, 1 \mathrm{H}), 7.63 \sim 7.59(\mathrm{~m}, 1 \mathrm{H}) ;{ }^{13} \mathrm{C} \mathrm{NMR}(75 \mathrm{MHz}$, $\left.\mathrm{CDCl}_{3}\right) \delta: 144.4,138.9,135.9,134.5,130.8,129.0,127.4$, $126.5,114.8,112.2$.

苯并 $[4,5]$ 咪唑 $[1,2-a]$ 喹喔啉 $(3 \mathrm{II})^{[12]}:$ m.p. $181 \sim 182$ ${ }^{\circ} \mathrm{C}$, 白色固体. ${ }^{1} \mathrm{H} \mathrm{NMR}\left(300 \mathrm{MHz}, \mathrm{CDCl}_{3}\right) \delta: 8.94(\mathrm{~s}$, $1 \mathrm{H}), 8.46 \sim 8.42(\mathrm{~m}, 2 \mathrm{H}), 7.40 \sim 7.36(\mathrm{~m}, 2 \mathrm{H}), 7.61(\mathrm{t}, J=$ $7.7 \mathrm{~Hz}, 1 \mathrm{H}), 7.55$ (t, $J=7.5 \mathrm{~Hz}, 1 \mathrm{H}), 7.46 \sim 7.41(\mathrm{~m}, 2 \mathrm{H})$; ${ }^{13} \mathrm{C}$ NMR (75 MHz, $\left.\mathrm{CDCl}_{3}\right) \delta: 146.0,144.3,141.1,135.6$, $131.0,129.9$, 129.8, 129.6, 125.8, 125.6, 124.8, 122.0, 114.8, 114.2.

9-甲基苯并 $[4,5]$ 咪唑[1,2- $a$ ]喹喔啉(3m): m.p. 171 $172{ }^{\circ} \mathrm{C}$, 白色固体. ${ }^{1} \mathrm{H} \mathrm{NMR}\left(300 \mathrm{MHz}, \mathrm{CDCl}_{3}\right) \delta$ : $8.88(\mathrm{~s}$, $1 \mathrm{H}), 8.26 \sim 8.20(\mathrm{~m}, 2 \mathrm{H}), 7.92(\mathrm{dd}, J=7.9,1.4 \mathrm{~Hz}, 1 \mathrm{H})$, $7.56 \sim 7.52(\mathrm{~m}, 1 \mathrm{H}), 7.47(\mathrm{dd}, J=8.9,2.6 \mathrm{~Hz}, 1 \mathrm{H}), 7.40 \sim$ $7.36(\mathrm{~m}, 1 \mathrm{H}), 7.30 \sim 7.23(\mathrm{~m}, 1 \mathrm{H}), 2.57(\mathrm{~s}, 3 \mathrm{H}) ;{ }^{13} \mathrm{C} \mathrm{NMR}$ $\left(75 \mathrm{MHz}, \mathrm{CDCl}_{3}\right) \delta: 148.0,136.0,132.7,131.0,130.5$, $129.8,129.2,128.7,124.4,124.2,123.0,122.7,114.9$, 114.6, 29.7; HRMS (EI) calcd for $\mathrm{C}_{15} \mathrm{H}_{11} \mathrm{~N}_{3}(\mathrm{M}+\mathrm{H})^{+}$ 234.1019, found 234.1023.

9-氯基苯并 $[4,5]$ 咪唑[1,2- $a$ ]喹喔啉(3n): m.p. 174 $176{ }^{\circ} \mathrm{C}$, 黄色固体. ${ }^{1} \mathrm{H} \mathrm{NMR}\left(300 \mathrm{MHz}, \mathrm{CDCl}_{3}\right) \delta: 8.88$ (s, $1 \mathrm{H}), 8.25 \sim 8.21(\mathrm{~m}, 2 \mathrm{H}), 7.93(\mathrm{dd}, J=7.9,1.4 \mathrm{~Hz}, 1 \mathrm{H})$, $7.56 \sim 7.52(\mathrm{~m}, 1 \mathrm{H}), 7.45(\mathrm{dd}, J=8.9,2.6 \mathrm{~Hz}, 1 \mathrm{H}), 7.40 \sim$ $7.36(\mathrm{~m}, 1 \mathrm{H}), 7.27 \sim 7.22(\mathrm{~m}, 1 \mathrm{H}) ;{ }^{13} \mathrm{C} \mathrm{NMR}(75 \mathrm{MHz}$, $\left.\mathrm{CDCl}_{3}\right) \delta: 148.0,135.0,132.7,131.0,130.4,129.9,129.2$, $128.7,124.4,124.2,122.9,122.7,114.9,114.6$; HRMS (EI) calcd for $\mathrm{C}_{14} \mathrm{H}_{8} \mathrm{~N}_{3} \mathrm{Cl}(\mathrm{M}+\mathrm{H})^{+}$254.0451, found 254.0454 .

2-甲基吡唑 $[1,5-a]$ 喹喔啉(3o $)^{[13]}$ : m.p. $85 \sim 88{ }^{\circ} \mathrm{C}$, 浅灰色固体. ${ }^{1} \mathrm{H}$ NMR $\left(300 \mathrm{MHz}, \mathrm{CDCl}_{3}\right) \delta: 8.94$ (s, 1H), $8.42(\mathrm{dd}, J=8.3,1.0 \mathrm{~Hz}, 1 \mathrm{H}), 8.03(\mathrm{dd}, J=8.1,1.0 \mathrm{~Hz}$, $1 \mathrm{H}), 7.67 \sim 7.63(\mathrm{~m}, 1 \mathrm{H}), 7.54 \sim 7.50(\mathrm{~m}, 1 \mathrm{H}), 6.63(\mathrm{~s}$,
1H), $2.58(\mathrm{~s}, 3 \mathrm{H}) ;{ }^{13} \mathrm{C} \mathrm{NMR}\left(75 \mathrm{MHz}, \mathrm{CDCl}_{3}\right) \delta: 151.7$, $143.8,135.7,133.8,129.8,129.5,129.3,125.9,114.7$, $101.1,14.1$.

\section{References}

[1] (a) Guillon, J.; Dumoulin, H.; Dallemagne, P.; Rault, S. Pharm. Pharmacol. Commun. 1998, 4, 33.

(b) Guillon, J.; Boulouard, M.; Rault, S. J. Pharm. Pharmacol. 2000, 52, 1369.

(c) Guillon, J.; Dallemagne, P.; Rault, S. Eur. J. Med. Chem. 1998, 33,293

(d) Gemma, S.; Colombo, L.; Tripaldi, P. Org. Biomol. Chem. 2011, 9, 5137.

[2] (a) Alleca, S.; Corona, P.; La Colla, P. Farmaco 2003, 58, 639. (b) Guillon, J.; Forfar, I.; Dufaure, B. Bioorg. Med. Chem. 2007, 15, 194.

(c) Guillon, J.; Grellier, P.; Péhourcq, F. J. Med. Chem. 2004, 47, 1997.

(d) Guillon, J.; Mouray, E.; Le-Naour, A. Eur. J. Med. Chem. 2011, 46, 2310.

(e) Guillon, J.; Moreau, S.; Jarry, C. Bioorg. Med. Chem. 2008, 16, 9133.

[3] (a) Morelli, E.; Gemma, S.; Budriesi, R.; Borrelli, G. J. Med. Chem. 2009, 52, 3548.

(b) Prunier, H.; Rault, S.; Guardiola-Lemaitre, B. J. Med. Chem. 1997, 40, 1808.

(c) Butini, S.; Budriesi, R.; Hamon, M.; Ioan, P. J. Med. Chem. 2009, 52, 6946.

[4] Cheeseman, G.; Tuck, B. Chem. Ind. 1965, 1382.

[5] Biswas, S.; Batra, S. Eur. J. Org. Chem. 2013, 4895.

[6] Yuan, Q.; Ma, D. J. Org. Chem. 2008, 73, 5159.

[7] Reeves, J. T.; Fandrick, D. R.; Senanayake, C. H. J. Org. Chem. 2010, 75, 992.

[8] Pereira, M. D. F.; Thiéry, V. R. Org. Lett. 2012, 14, 4754

[9] Huang, A. P.; Chen, Y. M.; Ma, C. Org. Lett. 2013, 21, 5480.

[10] (a) Yin, H. Q.; Jin, M.; Chen, W.; Chen, C.; Zheng, L. K.; Wei, P.; Han, S. Q. Tetrahedron Lett. 2012, 53, 1265.

(b) Zhang, J. X.; Yin, H. Q.; Han, S. Q. Chin. J. Org. Chem. 2012, 32, 1429 (in Chinese).

(张敬先，殷慧清，韩世清，有机化学， 2012, 32, 1429.)

(c) He, G. Z.; Huang, Y.; Tong, Y.; Han, S. Q. Tetrahedron Lett. 2013, 54, 5318 .

(d) Huang, Y.; Chen, C.; Zhao, D.; Han, S. Q. Chin. J. Chem. 2013, $31,1007$.

(e) Jin, M.; Zhao, D.; He, G. Z.; Han, S. Q. Chin. J. Catal. 2013, 34, 1651.

[11] Ste'phanie, P.; Parra, S. Eur. J. Med. Chem. 2001, 36, 255.

[12] Bergman, J.; Vallberg, H. Acta Chem. Scand. 1997, 51, 742.

[13] Albini, A.; Bettinetti, G.; Minoli, G. J. Am. Chem. Soc. 1997, 119, 7308. 